\title{
Lesões cranianas por Projéteis de Arma de Fogo (PAF): possíveis efeitos do chumbo
}

\author{
Cranial injuries by Fire Weapon Projectors (FWP): possible effects of lead \\ Lesiones craneales por Proyectores de Armas de Fuego (PAF): posibles efectos del \\ plomo
}

Daniel Lopes Marques de Araújo ${ }^{1 *}$, Daniel Vinicius Eloi ${ }^{1}$, Gonzaga de Souza Gontijo Júnior ${ }^{1}$, Isabelle

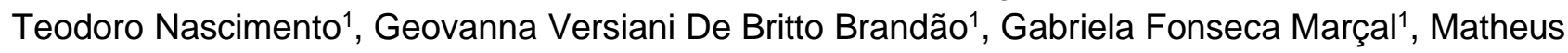
Garcia Ribeiro', Talitha Araújo Velôso Faria'.

\section{RESUMO}

Objetivo: Apresentar os possíveis efeitos tóxicos do chumbo em vítimas de traumatismo cranioencefálico por Projéteis de Arma de Fogo (PAF) suas principais manifestações clínicas, visando uma intervenção precoce e eficaz. Revisão bibliográfica: A intoxicação por chumbo é uma complicação tardia do trauma por PAF, decorrente do processo de cavitação. Também conhecida como saturnismo, ela consiste no acúmulo desse metal em um organismo, sendo o principal fator de risco o contato do projétil com o Líquido Cefalorraquidiano (LCR), que possui um pH ácido responsável por solubilizar o chumbo, que entra na corrente sanguínea e faz lesões em órgãos-alvo, como neurodegeneração à nível encefálico. A principal forma de tratamento é a remoção cirúrgica dos fragmentos de projétil, no entanto é importante se atentar à quantidade, distribuição e áreas afetadas, antes de realizar o procedimento. Considerações finais: Entre as complicações das lesões cranianas por PAF, a intoxicação por chumbo é um dos diagnósticos menos cogitados, no entanto não menos importante. Esta condição pode evoluir com danos neurológicos e sistêmicos que comprometem a saúde dos pacientes, sendo de grande relevância o diagnóstico precoce e intervenção, para prevenir agravamentos.

Palavras-chave: Ferimentos por arma de fogo, Intoxicação por chumbo, Trauma craniocerebral.

\section{ABSTRACT}

Objective: To present the possible toxic effects of lead in victims of traumatic brain injury due to Fire Weapon Projectors (FWP) and its main clinical manifestations, aiming at an early and effective intervention. Bibliographic review: Lead poisoning is a late complication of trauma by PAF, resulting from the cavitation process. Also known as saturnism, it consists of the accumulation of this metal in an organism, the main risk factor being the contact of the projectile with the Cerebrospinal Fluid (CSF), which has an acidic $\mathrm{pH}$ responsible for solubilizing lead, which enters the bloodstream and causes damage to organs target, such as neurodegeneration at the brain level. The main form of treatment is the surgical removal of the projectile fragments, however it is important to pay attention to the quantity, distribution and affected areas, before performing the procedure. Final considerations: Among the complications of cranial injuries caused by PAF, lead poisoning is one of the least considered diagnoses, however no less important. This condition can evolve with neurological and systemic damage that compromise the health of patients, with early diagnosis and intervention being of great importance to prevent worsening.

Key words: Gunshot wounds, Lead poisoning, Craniocerebral trauma.

\section{RESUMEN}

Objetivo: Presentar los posibles efectos tóxicos del plomo en víctimas de traumatismo craneoencefálico por Proyectores de Armas de Fuego (PAF) y sus principales manifestaciones clínicas, con el objetivo de una intervención temprana y eficaz. Revisión bibliográfica: El envenenamiento por plomo es una complicación

\footnotetext{
${ }^{1}$ Centro Universitário Atenas (UniAtenas), Paracatu - MG. *E-mail: danielmedaraujo@gmail.com
} 
tardía del trauma por PAF, resultante del proceso de cavitación. También conocido como saturnismo, consiste en la acumulación de este metal en un organismo, siendo el principal factor de riesgo el contacto del proyectil con el Fluido Cerebroespinal (LCR), el cual tiene un pH ácido responsable de solubilizar el plomo, que ingresa al torrente sanguíneo y causa daño a los órganos objetivo, como la neurodegeneración a nivel cerebral. La principal forma de tratamiento es la remoción quirúrgica de los fragmentos del proyectil, sin embargo es importante prestar atención a la cantidad, distribución y áreas afectadas, antes de realizar el procedimiento. Consideraciones finales: Entre las complicaciones de las lesiones craneales causadas por la PAF, la intoxicación por plomo es uno de los diagnósticos menos considerados, pero no menos importante. Esta patología puede evolucionar con daños neurológicos y sistémicos que comprometan la salud de los pacientes, siendo de gran importancia el diagnóstico e intervención precoces para evitar su agravamiento.

Palabras clave: Lesiones por arma de fuego, Envenenamiento por plomo, Trauma craneoencefálico.

\section{INTRODUÇÃO}

Todos os anos, 1,3 milhão de indivíduos falecem no mundo devido à violência. Tendo em vista somente os homicídios, a América Latina mostra os maiores números e o Brasil aparece como o oitavo país mais violento a nível mundial. Espancamentos, ferimentos por arma branca e arma de fogo são alguns exemplos de atos de natureza física, muito presentes no país, que configuram violência interpessoal. O uso de armas de fogo e também a posse são fatores que contribuem para os altos níveis de violência (MIRACLE VA, 2017).

No Brasil, mais de 1 milhão de homicídios foram contabilizados entre 1996 e 2016. Somente em 2016, 62.517 pessoas foram mortas, sendo que cerca de $70 \%$ foram acarretadas por ferimentos por arma de fogo. Anualmente, no país, meio milhão de pessoas requerem hospitalização devido a esses traumas. Eles representam, entre as internações por causas externas, a maior taxa de mortalidade, com aproximadamente 10 óbitos por 100 internações. O número de mortes por essa lesão é superado apenas pelas neoplasias e doenças cardiovasculares (BARROS JP, et al., 2020).

Os ferimentos por arma de fogo podem causar diversos efeitos deletérios nas vítimas que sofrem esse tipo de lesão. Tendo em vista que em várias situações o projétil pode ficar alojado no corpo, efeitos nocivos além das lesões traumáticas precisam ser investigados. A literatura médica descreve situações em que as balas ficam depositadas em diversas regiões corpóreas, contudo existem poucos estudos que elucidam essa situação envolvendo a região cranioencefálica (CAMPOS JS, et al., 2021).

As balas possuem em sua composição uma grande quantidade de chumbo (Pb) que ao entrar em contato com a corrente sanguínea pode causar sérias complicações para a saúde. Sob essa ótica, lesões cranioencefálicas por arma de fogo possuem potencial nocivo ao sistema neurológico. Ao ficarem alojadas no crânio dos indivíduos, as balas podem causar um tipo de dano chamado intoxicação por chumbo. Esse metal pesado tem a capacidade de interagir com as células nervosos e causar neurodegeneração com intensas repercussões clínicas. Dessa forma, tal patologia precisa ser estudada e seus efeitos nocivos necessitam ser compreendidos de forma mais ampla (ANDRÉ M, et al., 2020).

O trauma causado pelos projéteis, principalmente na região craniana, pode resultar em diversas alterações fisiopatológicas na vítima, como a intoxicação por chumbo, um agravamento pouco evidenciado na prática clínica. A clínica dessa patologia envolve debilidades neurológicas, e a intervenção cirúrgica precoce é necessária para evitar o agravamento desses danos. Com isso, é necessário apresentar os efeitos do chumbo no organismo humano e reunir os principais achados clínicos, em vista de uma intervenção precoce e uma conduta mais adequada. A intervenção cirúrgica para retirar o projetil evita a liberação do chumbo no organismo, e sua retirada precoce traz benefícios ao paciente (TRINDADE RFC, et al., 2015; MAIA BG, et al., 2013).

Nesse contexto, o estudo em questão visa retratar os possíveis efeitos fisiológicos causados pelo $\mathrm{Pb}$ no organismo de indivíduos com traumatismo cranioencefálico resultante de Projéteis de Arma de Fogo (PAF) e as consequências da intoxicação de chumbo em vítimas por arma de fogo. 


\section{REVISÃO BIBLIOGRÁFICA}

\section{Balística e capacidade destrutiva do projétil}

A priori, antes de explanar sobre os possíveis efeitos do chumbo em lesões cranianas por PAF, é válido elucidar os danos físicos causados pelos projéteis. A balística é uma área científica que estuda o movimento de um projétil levando em consideração o seu trajeto no cano da arma (balística interna), o seu trajeto no ar (balística externa) e o seu percurso no alvo (balístico terminal). A capacidade de os projéteis causarem lesão está relacionada a sua energia cinética (VAKIL MT e SINGH AK, 2017).

De acordo com a fórmula da energia cinética, um aumento na velocidade acarreta um ganho exponencial na energia, assim, a velocidade impacta mais significativamente o poder lesivo do projétil do que a sua massa. Os projéteis que alcançam menos que $300 \mathrm{~m} / \mathrm{s}$ são considerados de baixa velocidade, aqueles que atingem entre $300 \mathrm{~m} / \mathrm{s}$ e $600 \mathrm{~m} / \mathrm{s}$ são classificados como de velocidade média e, por fim, os que ultrapassam $600 \mathrm{~m} / \mathrm{s}$ são considerados de alta velocidade. As armas de mão são, em geral, de baixa e média velocidade, já as carabinas e espingardas são de média a alta velocidade, pois o cano mais longo permite à bala mais tempo para acelerar (ANTÃO KL, et al., 2019).

As balas podem causar danos nos tecidos por três maneiras diferentes: laceração e esmagamento, ondas de choque e cavitação. Os projéteis de baixa velocidade, como aqueles usados em pistolas e revólveres, provocam, geralmente, laceração e esmagamento dos tecidos por onde a bala faz o seu trajeto. Já os projéteis de alta velocidade causam ondas de choque e cavitação. A distensão temporária e rápida dos tecidos adjacentes ao percurso da bala é denominada cavitação. As ondas de choque comprimem e expandem os tecidos laterais e à frente da bala, além de criar um vácuo que pode trazer corpos estranhos para o ferimento. Devido a esses fenômenos, o orifício de saída da bala é maior que o de penetração e a magnitude desses danos está associada à energia cinética liberada pela bala (CAMPOS JS, et al., 2021).

Com projéteis de baixa velocidade, o impacto resultante pode gerar uma fratura no crânio em casos de lesão por arma de fogo, além disso, a onda de choque gerada pode causar hematomas e hemorragias em menor grau. No caso da cavitação, a expansão temporária do tecido cerebral, que consegue alcançar 30 vezes o diâmetro do projétil, pode pressioná-lo contra o crânio, causando morte por esmagamento do cérebro. Por outro lado, o vácuo criado pelas balas de alta velocidade promove a sucção de pele, cabelos, roupas e partículas do ambiente, contribuindo para a contaminação da área atingida. Ademais, as cavitações permanentes deixadas pelo percurso da bala no cérebro, tanto de baixa quanto de alta velocidade, podem acarretar intoxicação por chumbo, uma condição clínica com repercussões clínicas significativas, entretanto de difícil diagnóstico (BARROS JP, et al., 2020).

\section{Abordagem da intoxicação por chumbo}

Também denominado de saturnismo, a intoxicação por chumbo devido a PAF retido é um problema clínico grave, todavia pouco averiguado no cotidiano médico, caracterizado pelo importante acúmulo do metal no organismo de forma aguda ou crônica. Os fatores de risco para essa ocorrência estão relacionados principalmente ao fato de o projétil estar em contato com o Líquido Cefalorraquidiano (LCR). O pH ácido do meio promove a solubilização do chumbo, com mobilização no plasma e consequente ação no órgão-alvo (MADUREIRA PR, et al., 2009; RIBEIRO AP, et al., 2017).

Grande parte dos casos de intoxicação por chumbo não são descobertos e tratados, uma vez que a evolução clínica é lenta. Contudo, a concentração sérica de chumbo tem seu pico em $38,1 \%$ dos pacientes no terceiro mês após a lesão. Os projéteis de arma de fogo têm um núcleo de chumbo com uma cobertura de metal que, geralmente, é uma liga de cobre. Esse núcleo plúmbico pode incluir uma inserção de aço para dar à bala melhor penetração no alvo rígido. As lesões causadas por eles são predominantemente penetrantes, envolvendo projéteis pequenos, densos e perfurantes, assim como há munições que se fragmentam ao entrar em contato com o crânio (JANDIAL R, et al., 2008).

O parênquima cerebral tem alto teor de água e é relativamente inelástico, o que o torna particularmente suscetível a danos nos tecidos do trauma penetrante. A alta pressão do projétil resulta diretamente em lesões 
por esmagamento e necrose celular ao longo da ponta da bala, criando um trato permanente. A energia liberada pelo projétil, com a colisão, causa expansão e posteriormente retração do tecido, criando uma cavidade temporária que depois se instala e forma um trato maior que o inicial. Como consequência, pode haver contusões ou interrupção de vasos sanguíneos adjacentes (VAKIL MT e SINGH AK, 2017).

As complicações após a lesão cerebral penetrante são classificadas como precoces, se presentes em tempo menor que uma semana, e tardias após esse período. As precoces incluem hemorragia, contusão cerebral, lesão vascular, infecção, lesão cerebral isquêmica, e edema cerebral, enquanto complicações tardias incluem hidrocefalia, vazamento de LCR, migração de corpos estranhos e intoxicação por chumbo (FARHAT HI, et al., 2012).

\section{Os efeitos do chumbo no organismo humano}

O tempo decorrido entre o acidente e os sintomas iniciais varia de três meses a um tempo indefinido, de acordo com a área do projétil que está em contato com os líquidos orgânicos (MADUREIRA PR, et al., 2009). Os projéteis são fragmentados especialmente se atingem tecidos rígidos, como dentes ou ossos, o que predispõe o aumento dos níveis séricos do elemento. Indivíduos com projéteis ou fragmentos alojados perto de um osso apresentaram níveis de chumbo no sangue $32 \%$ mais altos que os indivíduos que não tinham (GONZÁLEZ-PÉREZ GJ, et al., 2017; MACIEL PR, et al., 2016).

No encéfalo, os efeitos do chumbo causam mudanças celulares que levam à neurodegeneração. $A$ excitotoxicidade do glutamato induzida pela lesão resulta na liberação de íons cálcio e na ativação de proteases dependentes de cálcio que iniciam a destruição citoesquelética. Com isso, há uma turgência das mitocôndrias juntamente à liberação de citocromo c no citosol, dissipação do potencial da membrana mitocondrial, aumento dos danos dos radicais livres, perda na produção de ATP e desequilíbrio iônico que culminam em necrose ou apoptose de neurônios (GAJAVELLI S, et al., 2015).

As manifestações clínicas mais usuais decorrentes da intoxicação são: gastrintestinais, como dor abdominal, vômitos e anorexia; hematológicas, como anemia normocrômica e normocítica ou anemia hipocrômica e microcítica; neurológicas, como cefaleia, ansiedade, depressão e irritabilidade; e insuficiência renal. A sintomatologia evidenciada é bastante inespecífica e pode ter início vários anos após o ferimento com o projétil, fazendo com que o diagnóstico de saturnismo seja tardio e, muitas vezes, nem cogitado. Até o ano de 2004, menos de 100 casos de toxicidade por chumbo em pacientes com projéteis retidos foram relatados na literatura médica, visto que o início dos sintomas é insidioso e exige um alto nível de suspeita para solicitar o teste. São pouco estudadas as condições associadas a níveis elevados de chumbo no sangue nesses pacientes (GOMES RCF, et al., 2015; PEARCE JMS, et al., 2007).

Um sinal semiológico específico do saturnismo é a linha de Burton, definida como uma coloração arroxeada na gengiva que é causada por uma reação entre o chumbo circulante com íons de enxofre liberados pela atividade bacteriana oral, que deposita sulfeto de chumbo na junção dos dentes e gengivas. $O$ sinal não é uma manifestação comum da intoxicação, porém, se presente, é uma pista clínica valiosa (PEARCE JMS, et al., 2007).

Ademais, vários estudos relatam o aumento da pressão arterial com uma elevação da carga corporal de chumbo. Sugere-se que a carga cumulativa de chumbo pode ser um fator de risco significativo para hipertensão. O chumbo no sangue acima de $40 \mu \mathrm{g} / \mathrm{dl}$ foi considerado o fator mais potente para elevar a pressão arterial sistólica e diastólica. Os dados sugerem que o chumbo induz mudanças no metabolismo das lipoproteínas que desempenham um papel importante no aumento da pressão arterial induzido pelo metal (D'SOUZA HS, et al., 2011).

Outra complicação que também pode ocorrer é a neuropatia periférica secundaria à intoxicação por chumbo, que possui uma clínica de fraqueza muscular generalizada, disfagia precoce, cefaloparestesia e parestesia nos membros superiores e inferiores, pode ter também atrofia e diminuição da força proximal. $O$ diagnóstico da miopatia inflamatória pode ser confirmada com base na elevação das enzimas musculares, inflamação muscular na ressonância magnética, com predominio de atrofia e biopsia. Os achados típicos em uma biópsia muscular, encontram sinias de miopatia, com aumento do diâmetro das fibras musculares, 
separados por um tecido conjuntivo, mitocôndrias hidrópicas e um sistema sarcotubular dilatado, com aumento de deposito extracelular e destruição nuclear (BECERRA L, et al., 2016).

O diagnóstico de saturnismo pode ser confirmado pela análise de chumbo no sangue ou pela medição de alguns dos parâmetros indiretos para o efeito do chumbo na síntese de hemoglobina, como ácido delta aminolevulínico (ALA) e protoporfirina de zinco, não obstante são pouco solicitados, uma vez que a intoxicação geralmente não é considerada como hipótese diagnóstica. Estudos relataram elevação na protoporfirina de zinco e uma diminuição no nível de hemoglobina em pessoas expostas a grandes quantidades de chumbo (BARROS JP, et al., 2020).

O chumbo inibe as enzimas ácido d-aminolevulínico desidratase, coproporfirinogênio oxidase e ferroquelataseda via de síntese do grupo heme. Como aferroquelataseé responsável pela incorporação de ferro na protoporfirina IX para formar heme, o resultado dessa inibição é o acúmuloda protoporfirinanos eritrócitos, com a maioria se ligando ao zinco e formando protoporfirina de zinco (D'SOUZA HS, et al., 2011; MADUREIRA PR, et al., 2009). Os fragmentos de projétil são frequentemente removidos cirurgicamente, a menos que a localização profunda deles impeça a remoção (VAKIL MT e SINGH AK, 2017).

O procedimento cirúrgico geralmente fornece tratamento preventivo e definitivo, contudo eventualmente tem pouco benefício, uma vez que pode colocar fragmentos do projétil em contato com o LCR e, como consequência, mobilizar o chumbo para a circulação sanguínea, promovendo sintomas de intoxicação do metal mesmo que o paciente ainda esteja assintomático. Os ciclos de terapia de quelação mostram grande eficácia durante os períodos sintomáticos. Eles reduzem os sintomas e sinais de envenenamento e promovem grande excreção de chumbo, reduzindo assim a carga total de chumbo responsável pelos sintomas (MADUREIRA PR, et al., 2009).

A terapia de quelação é conhecida por reduzir agudamente as concentrações de chumbo no sangue, ela aumenta a taxa de excreção do metal no curto prazo, em até 25-30 vezes o normal, o que caso contrário, pode levar de meses a anos. Entretanto, os níveis podem se elevar dentro de semanas a meses após o tratamento, exigindo, de forma frequente, ciclos repetidos de tratamento (D'SOUZA HS, et al., 2011).

A migração intracraniana de projéteis é uma complicação incomum, embora potencialmente significativa. Ela pode colocar fragmentos do projétil em contato com o LCR e, consequentemente, mobilizar o chumbo na circulação sanguínea, causar alterações no exame neurológico do paciente. A migração também pode implicar no desenvolvimento de uma complicação intracraniana, como abscesso intracraniano, assim como pode ocorrer em um hematoma em evolução, dentro do sistema ventricular ou após a cirurgia. As complicações tardias podem resultar em convulsões, hematomas e hidrocefalia obstrutiva. Dependendo da localização do fragmento, a cirurgia pode ser indicada para fragmentos migrados. A quantidade, a distribuição de fragmentos e o comprometimento ou não de áreas significativas dificultam a definição dos benefícios de uma intervenção cirúrgica (TRINDADE RFC, et al., 2015;VAKIL MT e SINGH AK, 2017).

\section{CONSIDERAÇÕES FINAIS}

Entre as complicações das lesões cranianas por PAF, a intoxicação por chumbo é um dos diagnósticos menos cogitados, no entanto não menos importante, uma vez que, alojado no encéfalo, o projétil sofre ação do LCR, cujo $\mathrm{pH}$ promove a solubilização do chumbo no plasma, o qual, com o tempo, desencadeia debilidades neurológicas e sistêmicas. A condição tem desenvolvimento lento e insidioso, dificultando a identificação da intoxicação pelo metal, o que evidencia a importância de trazer o tema à tona, como neste trabalho. A intervenção mais utilizada para evitar a complicação é a remoção cirúrgica do projétil, entretanto alguns fatores como quantidade, distribuição de fragmentos e áreas atingidas devem ser levados em consideração.

\section{REFERÊNCIAS}

1. ANDRÉ M, et al. Ferimento em tórax com arma branca oculta na lesão : relato de caso. Revista Eletrônica Acervo Saúde, 2020; Sup(45): e3221. 
2. ANTÃO KL, et al. Perfil epidemiológico de vítimas de violência atendidos em hospital de emergência. Revista Eletrônica Acervo Saúde, 2019; 11(10): e395.

3. BARROS JP, et al. Desafios da assitência de enfermagem aos indivíduos vítimas de violência por armas de fogo e branca. Revista Eletrônica Acervo Saúde, 2020; 12(12): e5045.

4. BECERRA L, et al. Co-existence of peripheral neuropathy secondary to lead poisoning and chronic polymyositis: Case report. Revista Colombiana de Reumatologia, 2016; 23(3): 213-7.

5. CAMPOS JS, et al. Correlação entre o tempo para a primeira sedestação beira leito e o tempo de internação hospitalar em vítimas de traumatismo cranioencefálico. Revista Eletrônica Acervo Saúde, 2021; 13(4): e6755.

6. D'SOUZA HS, et al. Diagnosis, evaluation, and treatment of lead poisoning in general population. Indian Journal of Clinical Biochemistry, 2011; 26(2): 197-201.

7. FARHAT HI, et al. A tangential gunshot wound to the head: Case report and review of the literature. Journal of Emergency Medicine, 2012; 43(2): e111-4.

8. GAJAVELLI S, et al. Glucose and oxygen metabolism after penetrating ballistic-like brain injury. Journal of Cerebral Blood Flow and Metabolism, 2015; 35(5): 773-80.

9. GOMES RCF, et al. Saturnism after accident by large caliber firearm: case report. Revista Médica de Minas Gerais, $2015 ; 25(1): 120-4$.

10. GONZÁLEZ-PÉREZ GJ, et al. El incremento de la mortalidad por armas de fuego y su relación con el estancamiento de la esperanza de vida en México. Ciência e Saúde Coletiva, 2017; 22(9): 2861-72.

11. JANDIAL R, et al. Ballistics for the neurosurgeon. Neurosurgery, 2008; 62(2): 472-80.

12. MACIEL PR, et al. Estudo descritivo do perfil das vítimas com ferimentos por projéteis de arma de fogo e dos custos assistenciais em um hospital da Rede Viva Sentinela. Epidemiologia e Serviços de saúde: Revista do Sistema Único de Saúde do Brasil, 2016; 25(3): 607-16.

13. MADUREIRA PR, et al. Lead poisoning due to gunshot bullet in contact with cerebrospinal fluid: Case report. São Paulo Medical Journal, 2009; 127(1): 52-4.

14. MAIA BG, et al. Perfil clínico-epidemiológico das ocorrências de traumatismo cranioencefálico. Revista Neurociencias, 2013; 21(1): 43-52.

15. MIRACLE VA. Lead Poisoning in Children and Adults. Dimensions of Critical Care Nursing, 2017; 36(1): 71-3.

16. PEARCE JMS. Burton's line in lead poisoning. European Neurology, 2007; 57(2): 118-9.

17. RIBEIRO AP, et al. Lesões provocadas por armas de fogo atendidas em serviços de urgência e emergência Brasileiros. Ciência e Saúde Coletiva, 2017; 22(9): 2851-60.

18. TRINDADE RFC, et al. Map of homicides by firearms: Profile of the victims and the assaults. Revista da Escola de Enfermagem, 2015; 49(5): 748-55.

19. VAKIL MT, SINGH AK. A review of penetrating brain trauma: epidemiology, pathophysiology, imaging assessment, complications, and treatment. Emergency Radiology, 2017; 24(3): 301-9. 